\title{
Section introduction \\ The potential of new technologies/approaches. Introduction to Sessions 3 and 4
}

Mitch Dowsett ${ }^{1,2}$

\author{
${ }^{1}$ Academic Department of Biochemistry, Royal Marsden NHS Trust, Fulham Road, London SW3 6JJ, UK \\ 2Breakthrough Breast Cancer Research Centre, Institute of Cancer Research, 237 Fulham Road, London SW3 6JB, UK
}

Corresponding author: Mitch Dowsett, mitch.dowsett@icr.ac.uk

Published: 18 December 2009

This article is online at

http://breast-cancer-research.com/supplements/11/S3/S9

The advances in biotechnology as applied to malignant disease, and to breast cancer in particular, have been astonishing over recent years. In the present section, four approaches of contemporary interest and importance were evaluated - particularly in relation to their potential for impacting on clinical management of the disease.

Circulating tumour cells are detected in more than one-half of patients with advanced breast cancer, and their prognostic significance above a threshold of five cells per sample has been clearly demonstrated in this setting. It is not yet clear whether this provides major benefits for management of the disease - but the potential for these cells to act as a material for biomarker assessment in trials of new therapeutics is substantial and is already being met in prostate cancer. In primary breast cancer the data are less compelling, with far fewer cells being found, providing a challenge for reproducible measurements in individuals. It is possible that noncellular markers in blood, particularly of circulating DNA, may act as improved markers of residual disease. The characterisation of DNA in an individual's tumour to identify specific aberrations and the use of that profile to create specificity to DNA in blood is an approach that is highly attractive and may become feasible in a few years as the comprehensive characterisation of tumour DNA becomes more affordable and sufficiently rapid (see below).

Over the past decade the greatest amount of biological investigation has been related to the RNA profiling of breast tumours. Seminal publications identifying so-called intrinsic subtypes have substantially influenced thinking about breast cancer subtypes. It is now widely agreed that this approach identified an important new set of tumours - described as basal tumours - which overlaps substantially with, but is not identical to, triple-negative tumours. Luminal A and luminal B subgroups of oestrogen receptor-positive tumours have also been described, but the separation of these two groups is much less defined with the biology being largely a continuum
Breast Cancer Research 2009, 11(Suppl 3):S9

(doi:10.1186/bcr2428)

(c) 2009 BioMed Central Ltd

from low proliferative luminal A tumours to highly proliferative luminal $B$ tumours. Many further signatures have been derived from the profiling of other sets of tumours, and some have resulted in the derivation of clinically applied multigene parameters - such as the Oncotype DX 21-gene score, and the Mammaprint 70-gene profile. Given the expenditure on these parameters, it is important that they now be compared with standard immunohistochemical approaches combined with classical clinical pathological features. RNA profiling is likely to be most valuable when it is applied thoughtfully to the assessment of specific bioclinical questions.

It is widely accepted that aberrations in DNA are the driving force behind malignancies. Technologies for assessing these have improved through a number of steps, such as comparative genomic hybridisation, both classical and array based, but the power of new/next-generation sequencing to identify essentially all differences between germline and tumour genotypes will provide enormous challenges. Harnessing the vast amount of data derived into meaningful information that can be linked to the biology and clinical outcome of tumours will be predictably problematic. While the $\$ 1,000$ genome is probably only a few years away, and this would make sequencing of DNA across tumours affordable, to create enormous catalogues of genomic information without giving substantial thought to study design is likely to be frustrating and of limited value.

The last presentation in this section (not reported fully in the present supplement) reports not just the use of protein changes using novel protein-based microarrays but also the integration of these changes with RNA and DNA analyses. Superficially this might be expected to complicate the linkage of data to clinical outcome yet further, given that even more aberrations will be identified. The key, however, is in the word integration - such that this should allow the significance of an aberration in one cellular fraction to be confirmed or refuted in another, and in particular functionality of the change to be 
established. This allows potential driver abnormalities to be distinguished from passengers. In addition, the linkage of such work to the cell line as well as tumours provides a greater opportunity to identify important biological processes and pathways as opposed to individual genes.

In conclusion, these technologies hold great promise for the future - but it is important that they should be applied thoughtfully to well-annotated clinical materials: to achieve the greatest value in clinical applicability.

\section{Competing interests}

The author declares that they have no competing interests.

\section{Acknowledgement}

This article has been published as part of Breast Cancer Research Volume 11 Suppl 3 2009: Controversies in Breast Cancer 2009. The full contents of the supplement are available online at http://breastcancer-research.com/supplements/11/S3. 